\title{
Characterisation of femtosecond laser inscribed long period gratings in photonic crystal fibre
}

\author{
Tom Allsop ${ }^{\mathrm{a}}$, Kyriacos Kalli ${ }^{\mathrm{b}}$, Kaiming Zhou ${ }^{\mathrm{a}}$, Graham Smith ${ }^{\mathrm{a}}$, Yicheng Lai ${ }^{\mathrm{a}}$, Michael Dubov ${ }^{\mathrm{a}}$, Kate \\ Sugden ${ }^{\mathrm{a}}$, David Webb ${ }^{\mathrm{a}}$, Ian Bennion ${ }^{\mathrm{a}}$, Michael Komodromos ${ }^{\mathrm{c}}$, \\ ${ }^{a}$ Photonic Research Group, Aston University, Aston Triangle, Birmingham, B4 7ET, UK; \\ banophotonics Research Laboratory, Cyprus University of Technology, Limassol, Cyprus; \\ ${ }^{c}$ Frederick Research Centre, 7 Filokyprou, Nicosia, Cyprus
}

\begin{abstract}
The use of high intensity femtosecond laser sources for inscribing fibre gratings has attained significant interest. The principal advantage of high-energy pulses is their ability for grating inscription in any material type without preprocessing or special core doping - the inscription process is controlled multi-photon absorption, void generation and subsequent local refractive index changes. The formation of grating structures in photonics crystal fibre has proven difficult, as the presence of holes within the fibre that allow wave-guidance impair and scatter the femtosecond inscription beam. Here we report on the consistent manufacture of long period gratings in endlessly single mode microstructure fibre and on their characterisation to external perturbations. Long period gratings are currently the subject of considerable research interest due to their potential applications as filters and as sensing devices, responsive to strain, temperature, bending and refractive index. Compared to the more mature fibre Bragg grating sensors, LPGs have more complex spectra, usually with broader spectral features. On the other hand they are intrinsically sensitive to bending and refractive index. Perhaps more importantly, the fibre design and choice of grating period can have a considerable influence over the sensitivity to the various parameters, for example allowing the creation of a bend sensor with minimal temperature cross-sensitivity. This control is not possible with FBG sensors. Here we compare the effects of symmetric and asymmetric femtosecond laser inscription.
\end{abstract}

Keywords: Femtosecond laser, long-period fibre gratings, temperature stability, polarisation dependence

\section{INTRODUCTION}

A fibre long period grating (LPG) is periodic refractive index variation inscribed axially along the core of an optical fibre. The inscription mechanism has been achieved using several means and can be induced by using ultra-violet irradiation (for photosensitive single-mode optical fibre, with an inscription mechanism dominated by photo-bleaching); through fusion arc or laser heating inscription (for non-photosensitive single-mode optical fibre, with an inscription mechanism dominated by compaction and structural change) or more recently by femtosecond lasers, where in the near infra-red (at 800-1100nm) the dominant inscription mechanism appears to be a combination of effects that include void creation, material compaction and the photo-elastic effect induced from thermal strain. Grating structures that have been inscribed using the aforementioned femtosecond technique are currently under investigation by several research groups $[1,2]$. As a result of the periodic refractive index variation along the length of the optical fibre, guided light is coupled out of the core region, where it interacts with a potentially infinite number of cladding modes. However, due to phase matching that occurs between the core and cladding modes this interaction occurs between discrete modes, producing a characteristic loss spectrum that also occurs at discrete wavelengths. The index modulation change within the core of a single mode optical fibre can vary depending upon the inscription method used, tending to be in the range $10^{-4}$ to 0.1 , and has a period for LPGs of typically between $100-600 \mu \mathrm{m}$. It is this index modulation that promotes coupling between the core and cladding modes and that produces a set of attenuation bands seen in the transmission spectrum of the optical fibre core mode.

Long period gratings are currently the subject of considerable research interest due to their potential applications as filters and as sensing devices [3]. This study of the LPG attenuation bands has yielded many potential applications in the

Photonic Crystal Fibers II, edited by Kyriacos Kalli, Waclaw Urbanczyk,

Proc. of SPIE Vol. 6990, 69900I, (2008)

0277-786X/08/\$18 $\cdot$ doi: $10.1117 / 12.781104$

Proc. of SPIE Vol. $6990699001-1$ 
field of sensing through the dependence of the position of the attenuation bands on strain $(\varepsilon)$, temperature $(T)$, the refractive index of the surrounding medium (ns) and bending [4-11]. The spectral shift of the attenuation band arises from the phase matching condition of the LPG [3]. In contrast to the more mature fibre Bragg grating sensors, LPGs are intrinsically sensitive to bending and refractive index. Perhaps more importantly, the fibre design and choice of grating period can have a considerable influence over the sensitivity to the various parameters, for example allowing the creation of a bend sensor with the potential for minimal temperature cross-sensitivity. This control is not possible with FBG sensors [12]. Whilst the sensitivity of LPGs is an advantage over fibre Bragg gratings, a general cross-sensitivity between parameters can cause problems, the most often being the aforementioned unwanted temperature sensitivity.

Recently considerable interest has been shown in using photonic crystal fibre (PCF) in conjunction with grating structures as fibre sensing devices; this is due to the "endless" geometric and material variations possible [13,14]. It has been shown [15] that LPGs in PCF can have very low temperature sensitivity thus reducing cross-sensitivity problems, resulting in a sensing system with greater resolution. One of the major problems to date is the inscription of the LPG into the PCF due to the fact that the majority of PCFs are made from pure fused-silica, which is not photosensitive to UV and thus traditional UV inscription techniques are not applicable. Several other inscription approaches have been used such as the electric-arc method [15], $\mathrm{CO}_{2}$ lasers [16] and femtosecond lasers [17] to overcome this problem with the femtosecond laser technique being recognised as possibly the most versatile. With the increasing usage of femtosecond lasers to fabricate LPGs in PCF, we feel it important to study the temporal spectral stability of such fibre devices, and have discovered that all the LPGs we produced exhibited post-fabrication spectral evolution at room temperature as well as at higher temperatures along with polarisation dependence, all of which seem to be related to the inscription power used.

In this paper we present a study of the dependence on curvature of the spectral characteristics of LPGs written into photonic crystal fibre using a femtosecond laser system. It is found that asymmetric (off-centre) inscription produces LPG attenuation bands that have a spectral sensitivity to the orientation of the curvature, producing red and blue wavelength shifts depending upon the bend direction and with low temperature sensitivity. A high dependence upon the polarisation state of the illuminating light is also observed. Here we compare the effects of symmetric and asymmetric femtosecond laser inscription on the location of the LPG absorption features, their polarisation dependences over extended time periods at room temperature and elevated temperatures and their sensitivity to fibre curvature.

\section{FABRICATION OF GRATING DEVICES}

Using Endlessly Single Mode (ESM) PCF from Crystal Fibre A/S, a series of LPGs with the same period (400 $\mu$ m) were fabricated with inscription pulse energies ranging from 410 to $650 \mathrm{~nJ}$, requiring various lengths to obtain maximum strength attenuation bands. The refractive index changes to create the LPG are introduced by a NIR femtosecond laser (800nm Spitfire/Evolution titanium/sapphire system, Spectra-Physics Lasers). The femtosecond laser radiation was focused at a predetermined point near the core with a $\times 100(\mathrm{NA}=0.55)$ microscope objective, while the fibre was moved in a direction parallel to the fibre axis. The translation speed was $100 \mu \mathrm{ms}^{-1}$. A cylindrical lens $(\mathrm{f}=100 \mathrm{~mm})$ was put in the proximity of the microscope objective to shape the laser beam at the point of inscription. The femtosecond laser system produced a $1 \mathrm{kHz}$ train of $150 \mathrm{fs}$ pulses at $800 \mathrm{~nm}$, see figure 1a, other details of the experimental set-up can be found elsewhere [17]. As a result of previous investigations into the spectral characteristics of femtosecond laser inscribed LPGs [18] the light output from a broadband source was polarised and then a polarisation controller was used to change the polarisation of the light illuminating the LPGs (see figure 1b). 


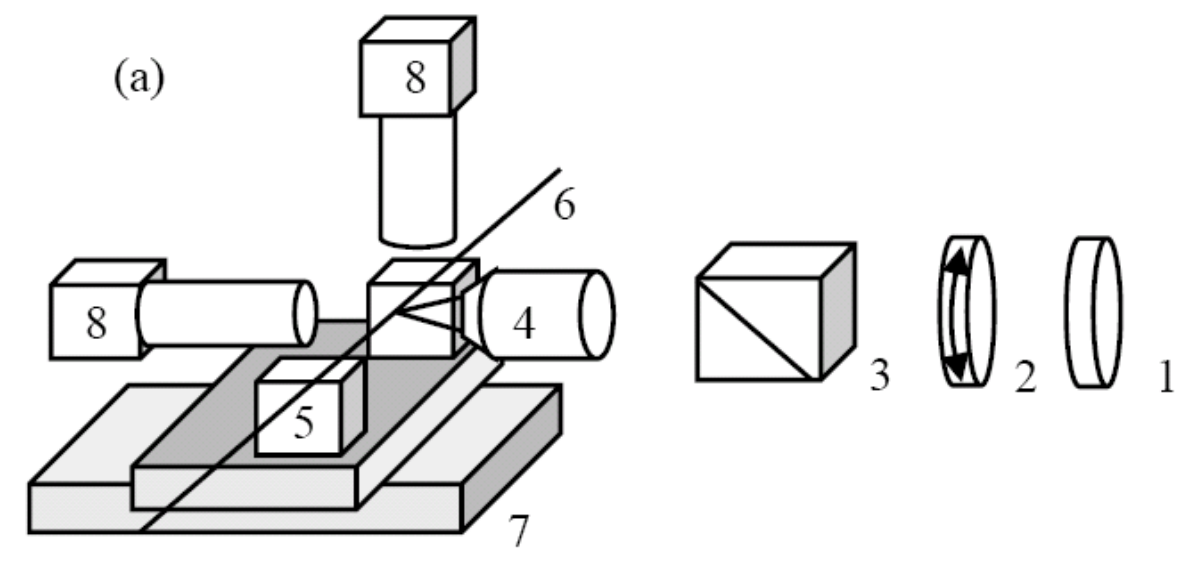

(b)

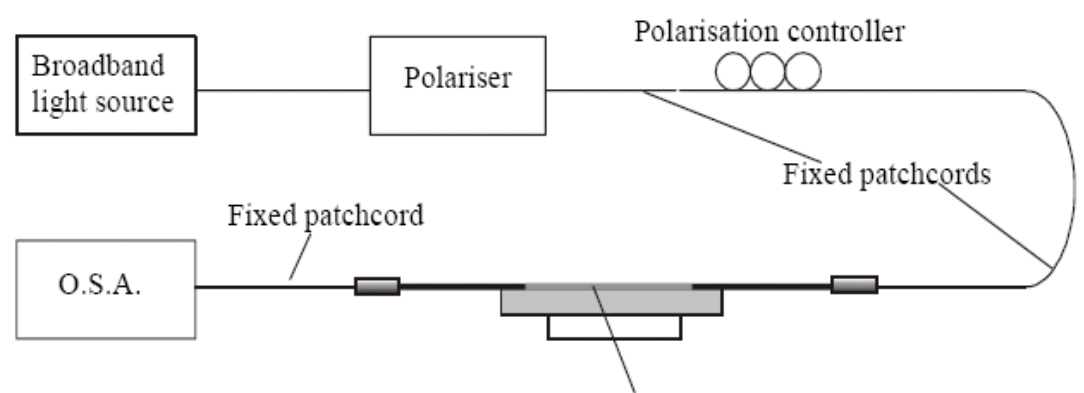

The Long Period Grating

Fig. 1. (a) Optical layout of inscription scheme. The light passes a shutter (1), a half-wave plate (2), Glan prism (3), x100 long working distance microscopic objective (4), before being focused in the core of the fibre (6). Two alignment $3 \mathrm{D}$ translation stages are mounted on top of the high precision computer controlled 2D-stage (7). Two CCD-cameras (8) are used with an optical zooming system for alignment and on-line monitoring of the inscription process. (b) A schematic of the apparatus used to investigate the transmission spectra of the LPGs during fabrication.

After inscription, the LPGs were examined under a microscope and the modified regions of the fibre were identified, as for example figure 2.

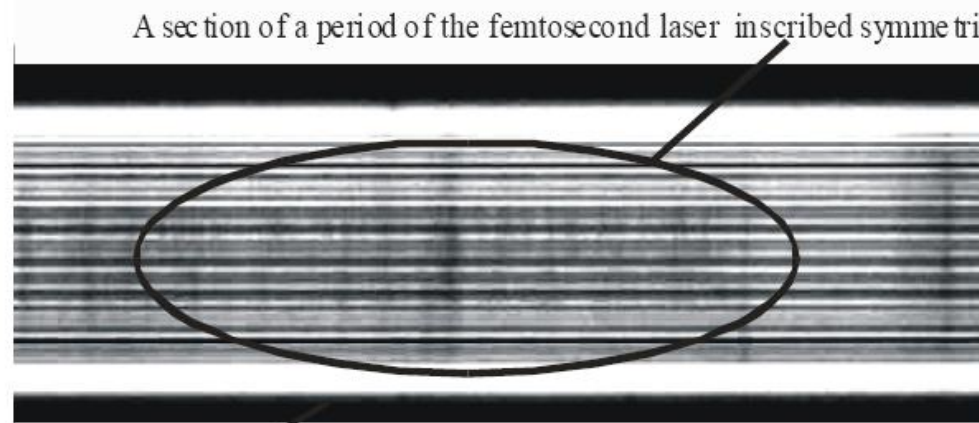

Endlessly single mode photonic crystal fibre

Fig.2. A photograph of part of a symmetrically inscribed section of a LPG in PCF 

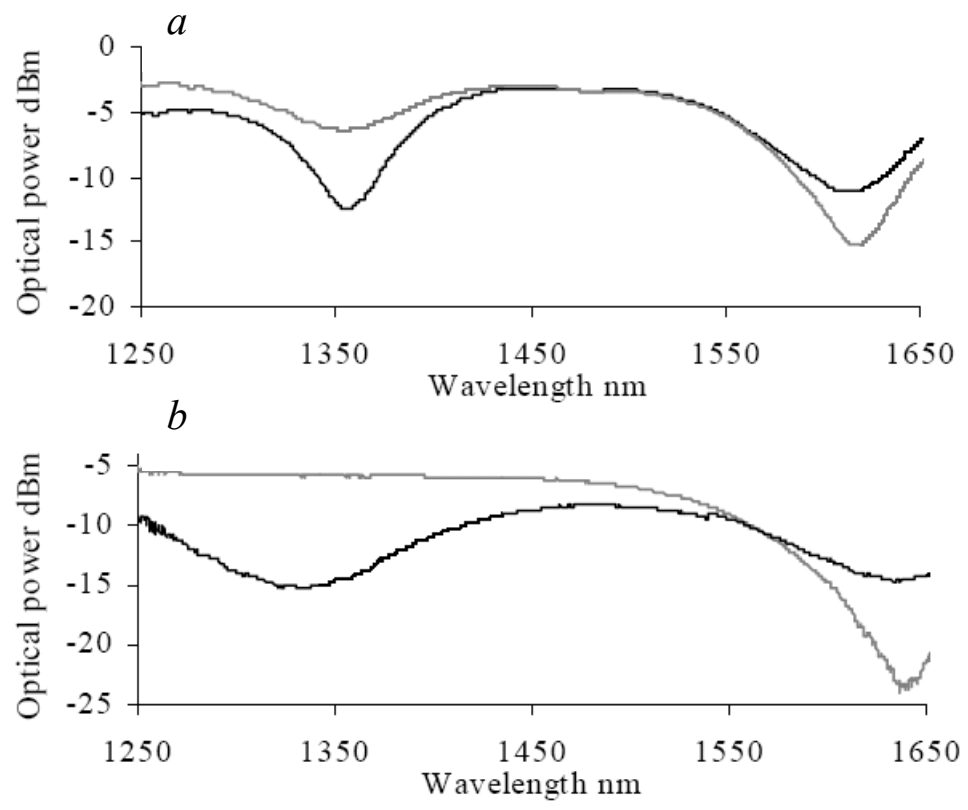

Fig. 3. Examples of transmission spectra of two LPGs (period $400 \mu \mathrm{m}$ ) fabricated in PCF (ESM) showing the polarisation dependence LPG spectra for (a) inscription energy $500 \mathrm{~nJ}$, grating length of $7 \mathrm{~mm}$. (b) inscription energy $650 \mathrm{~nJ}$, grating length of $4 \mathrm{~mm}$

The polarisation was constantly optimised to maximise coupling of the illuminating light into one of the attenuation bands present in the transmission spectrum of the LPG to observe the growth of the LPGs' attenuation bands during fabrication. Two examples of the transmission spectra are shown in figure $3 \mathrm{a}$ and $3 \mathrm{~b}$.

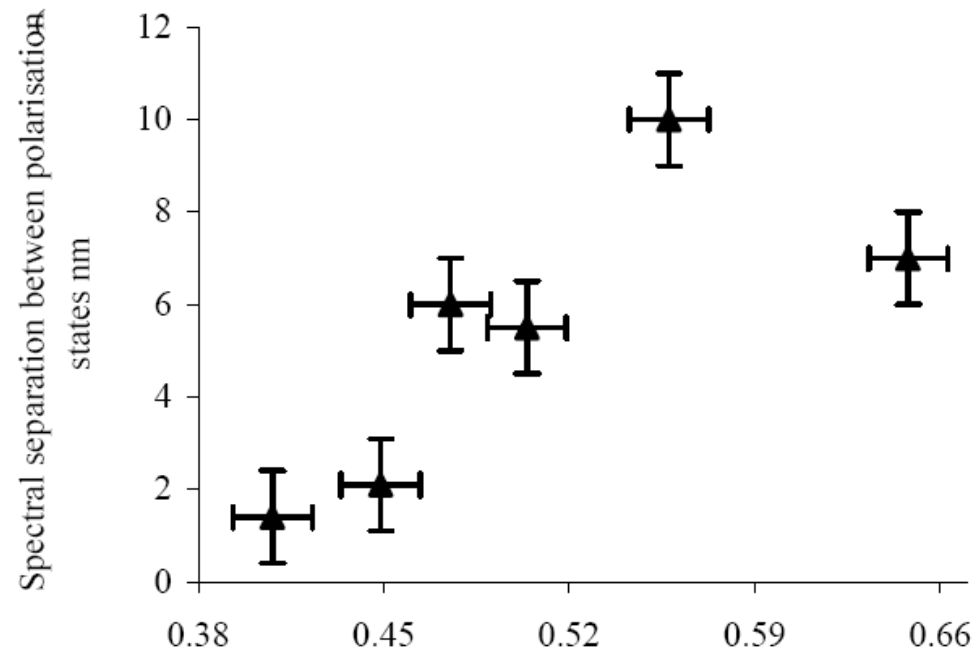

Inscription power $\mu \mathrm{J}$

Fig. 4. The maximum observed wavelength separation of the attenuation band due to polarisation as a function of inscription energy 
The polarisation dependence appears to have two main contributions one relating to the symmetry of the inscription and the other to the power of inscription. The birefringence ranged from $10.2 \mathrm{~nm}$ to $1.4 \mathrm{~nm}$ over the power range used, see figure 4.

Two further examples of the transmission spectra of femtosecond laser inscribed LPGs are shown in figure 5; one where the structural modification of the PCF appears to be radially symmetric about the fibre axis and the other where the structural modification is asymmetric, being displaced from the fibre axis (this being achieved by translating the fibre along the optical axis of the inscribing beam). The plots show the variation of the spectra obtained by adjusting the polarisation of the illuminating light to obtain the strongest coupling response to each of the attenuation bands present in the spectrum. The variations in resonant wavelength between the two polarisation states for the attenuation band close to $1590 \mathrm{~nm}$ were $1.4 \mathrm{~nm}$ for the symmetrically written LPG and $6.4 \mathrm{~nm}$ for the asymmetric one with figures of $0.5 \mathrm{~nm}$ and $4.1 \mathrm{~nm}$, respectively, for the stop bands at 1350nm.
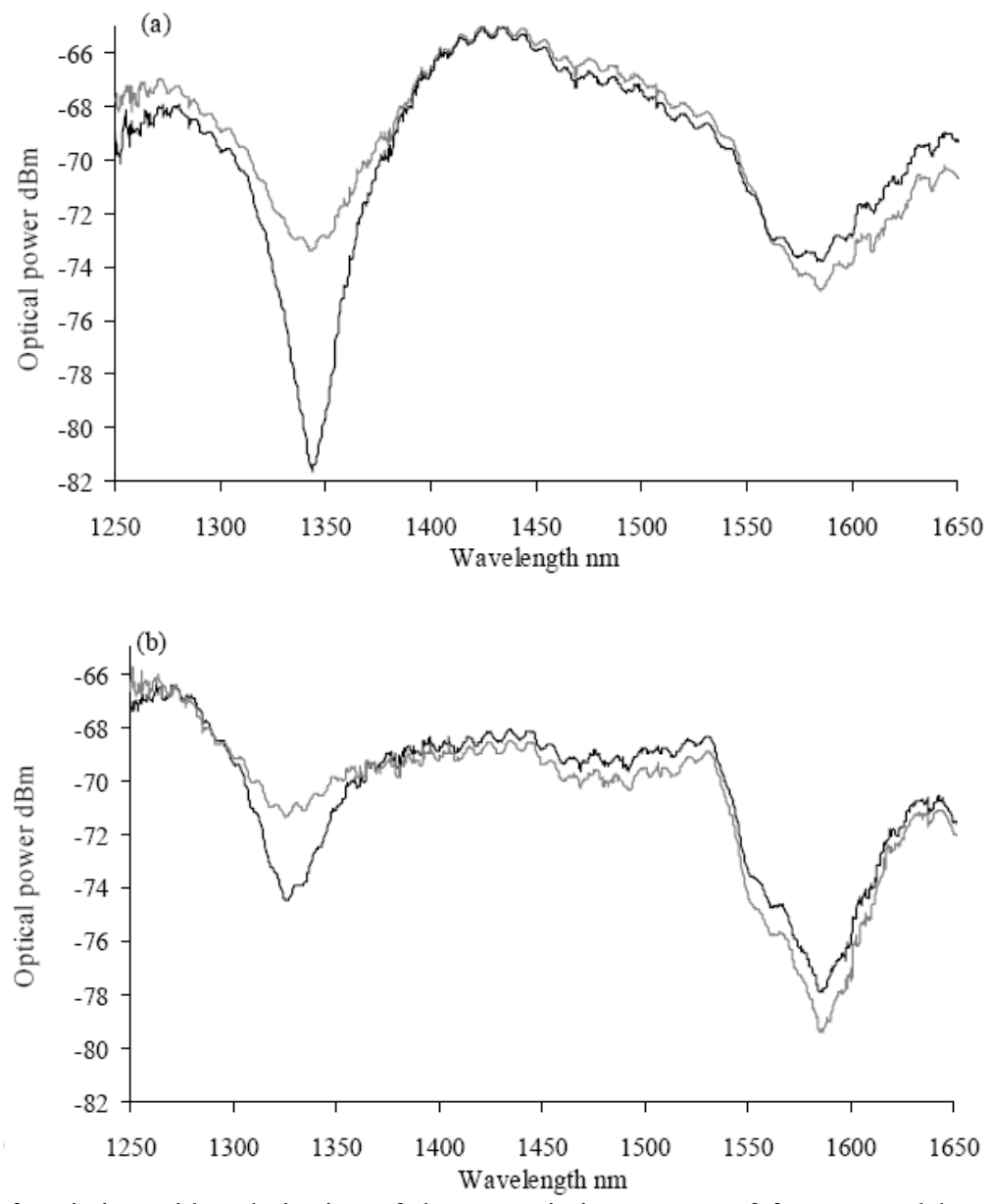

Fig. 5. Examples of variation with polarisation of the transmission spectra of femtosecond laser inscribed LPGs (period $400 \mu \mathrm{m})$ in photonic crystal fibre (a) asymmetrical inscription $(410 \mathrm{~nJ})$ (b) symmetrical inscription $(470 \mathrm{~nJ})$ 


\section{CURVATURE SENSITIVITY}

The LPG device was clamped between two towers; one of the clamps being mounted on a translation stage, which was moved inwards to induce a bend in the LPG placed mid-way between the clamps. Tags were used to ensure there was no twist in the fibre during the experiment and also to know the orientation of the LPG during bending, see figure 6 . The bend sensitivities of these LPGs were investigated using a broadband light source and a polariser, which in turn was connected to a polarisation controller; the light from this arrangement illuminated the LPGs and observations of the transmitted spectrum were made using an OSA. The polarisation controller was used to maximise coupling of the illuminating light into one of attenuation bands present in the transmission spectrum of the LPG, so one attenuation band could be studied at a time.

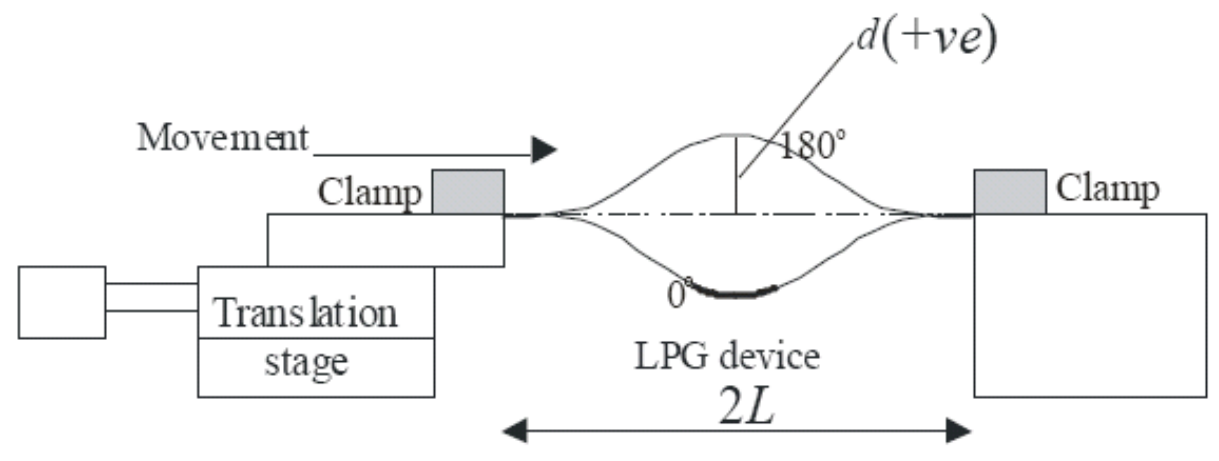

Fig. 6. Curvature test rig.

The sensor's curvature, $R$, is given by

$$
R=\frac{2 \cdot d}{\left(d^{2}+L^{2}\right)}
$$

where $L$ is the half distance between the edges of the two towers and $d$ is the bending displacement at the centre of the LPG. It was found from a microscopic inspection of the LPGs that the location of the femtosecond laser induced structural modification dramatically changed the spectral sensitivity and characteristics of the LPG. The symmetrically inscribed gratings exhibited no dependence of bend sensitivity on fibre orientation (see figure 7 for an example from the grating examined in figure $5 b$ ), while asymmetrically inscribed LPGs did display directional bend sensitivity of some attenuation bands; the transmission spectra of such a LPG (that used for figure 5a) is shown in figure 8, and the spectral sensitivities of the two attenuation bands are shown in figure 9. 

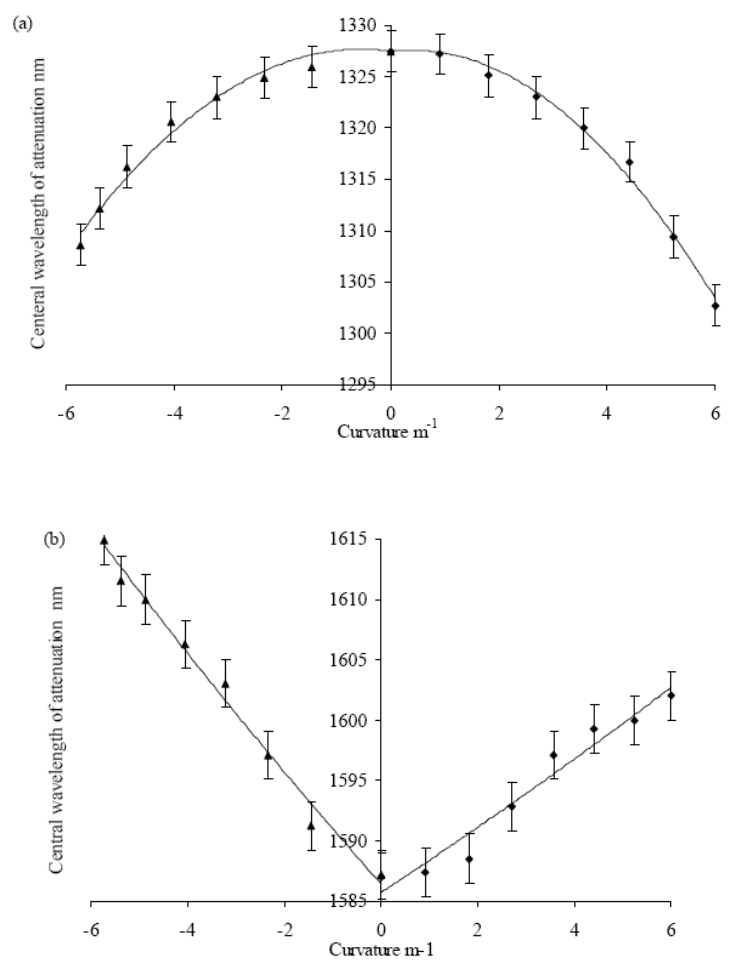

Fig. 7. (a) and (b) the spectral sensitivity to curvature of both observed attenuation bands of a symmetrically inscribed LPG in ESM PCF (period $=400 \mu \mathrm{m}$, length $=10 \mathrm{~mm}$, inscription energy $=470 \mathrm{~nJ}$ ).
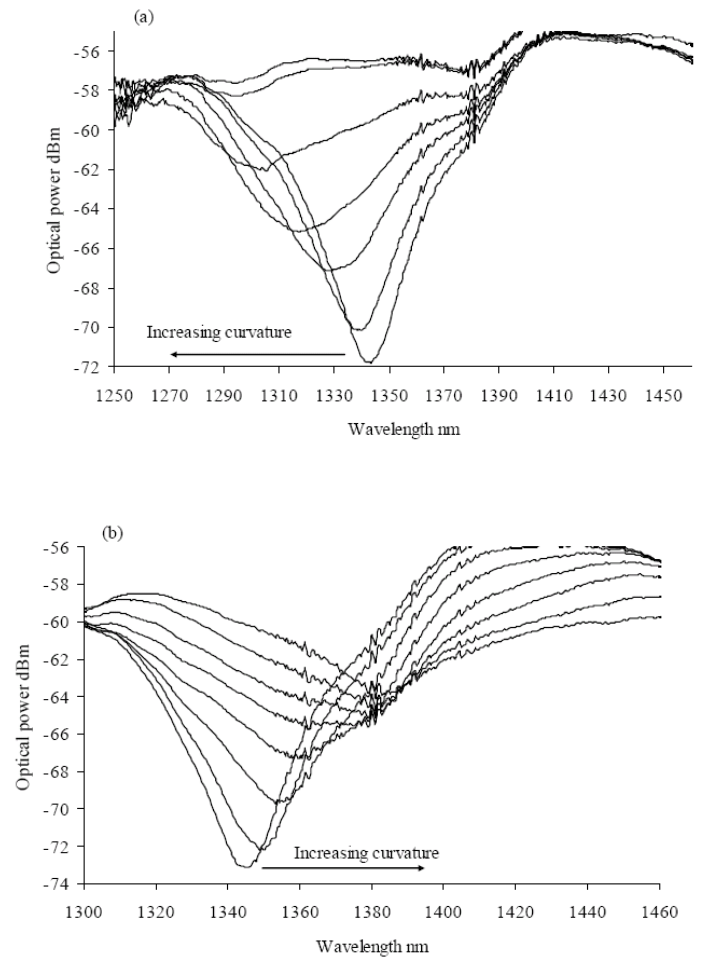

Fig. 8. An example of the curvature response of the transmission spectra of an asymmetrically inscribed LPG written in ESM PCF (period $=400 \mu \mathrm{m}$, length $=96 \mathrm{~mm}$, inscription energy $=410 \mathrm{~nJ}$ ). 

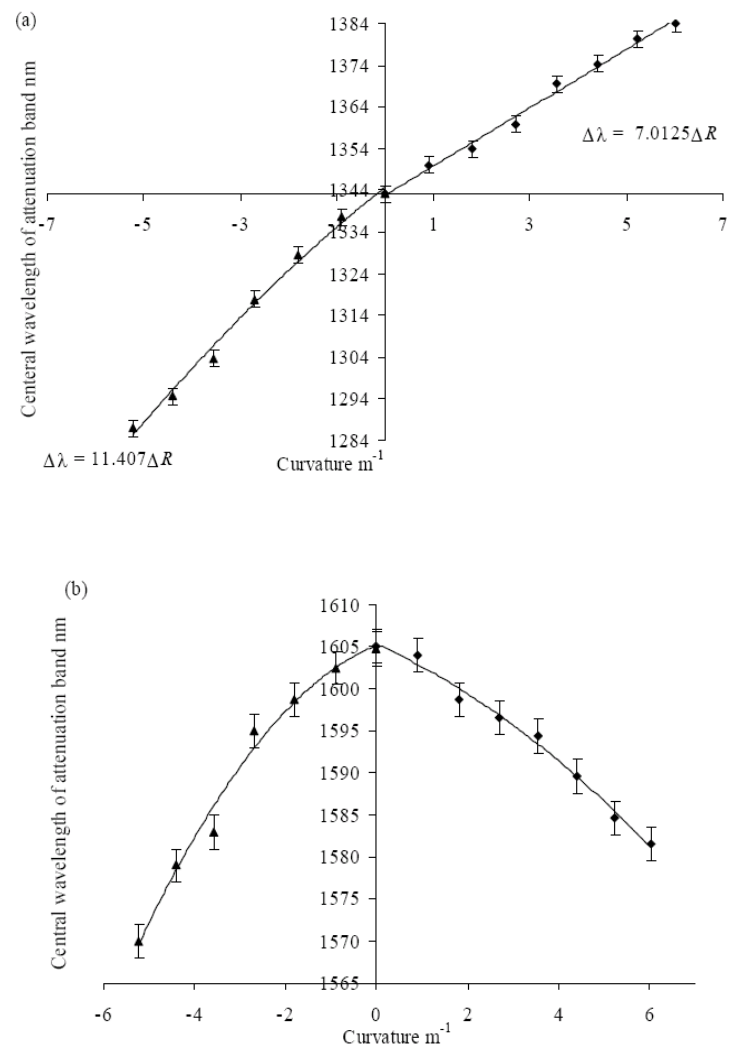

Fig. 9. (a) and (b) the spectral sensitivity to curvature of both observed attenuation bands of an asymmetrically inscribed LPG in ESM PCF (period $=400 \mu \mathrm{m}$, inscription energy $=410 \mathrm{~nJ}$. length $=9.6 \mathrm{~mm})$.

The asymmetrically inscribed LPGs in the PCF generate some attenuation bands which display a clear directional sensitivity (figure 9a), whilst others are much closer to giving a symmetrical response (figure 9b). The E-field profiles of the modes in this PCF have been investigated [12] and show dramatic variations in the radial distribution of the E-field with mode order, implying that we can expect radically different behaviour from the various modes. All the LPGs exhibited polarisation dependence with spectral birefringence ranging from 1 to $10 \mathrm{~nm}$, but this appears to be mainly due to the inscription method and the power used to fabricate the LPGs. The temperature sensitivity of these LPGs was also investigated; it was found to be $4.5 \times 10^{-3} \mathrm{~nm}^{\circ} \mathrm{C}^{-1}$ which is in approximate agreement with other workers [12] and considerably less than for LPGs written in SMF $[5,18]$.

\section{ANNEALING}

Annealing studies were carried out at room temperature and $80^{\circ} \mathrm{C}$; these temperatures were selected due to the fact that they are commonly used for the annealing of gratings in standard SMF. The experimental arrangement for measurements taken at room temperature is shown in figure $1 \mathrm{~b}$; at $80^{\circ} \mathrm{C}$ the LPGs were placed into an insulated box with a Peltier cooler.

\subsection{Annealing studies at room temperature}

At room temperature it was observed that LPGs had post-fabrication spectral evolution. For all observed LPGs the general trends appeared to be the same with an initial red wavelength shift followed by a further blue wavelength shift. The largest spectral shifts occurred in the first 120 hours of fabrication with the maximum observed wavelength shifts linked with the LPGs inscribed with the lowest powers. An example of the temporal response of the LPGs' transmission 
spectra is shown in figure 10a with the wavelength shift shown in figure 10b. For all LPGs observed at room temperature, the maximum wavelength shift observed is shown in figure 10c (note that the two results in the circle are measurements not taken at the same time as the other LPGs; one being at only 90 hours and the other at 147 hours after inscription). The other LPGs were measured at approximately 120 hours. After approximately 600 hours of room temperature annealing, the LPGs appeared to be spectrally stable but there were red and blue wavelength shifts compared to the resonant wavelength immediately following fabrication, which appear to be related to the inscription energy, see figure 11. Also there were variations observed in the polarisation induced wavelength shift of both bands, which appear to be related to both the energies used for inscription and how symmetric the inscription was, see figure $11 \mathrm{~b}$.
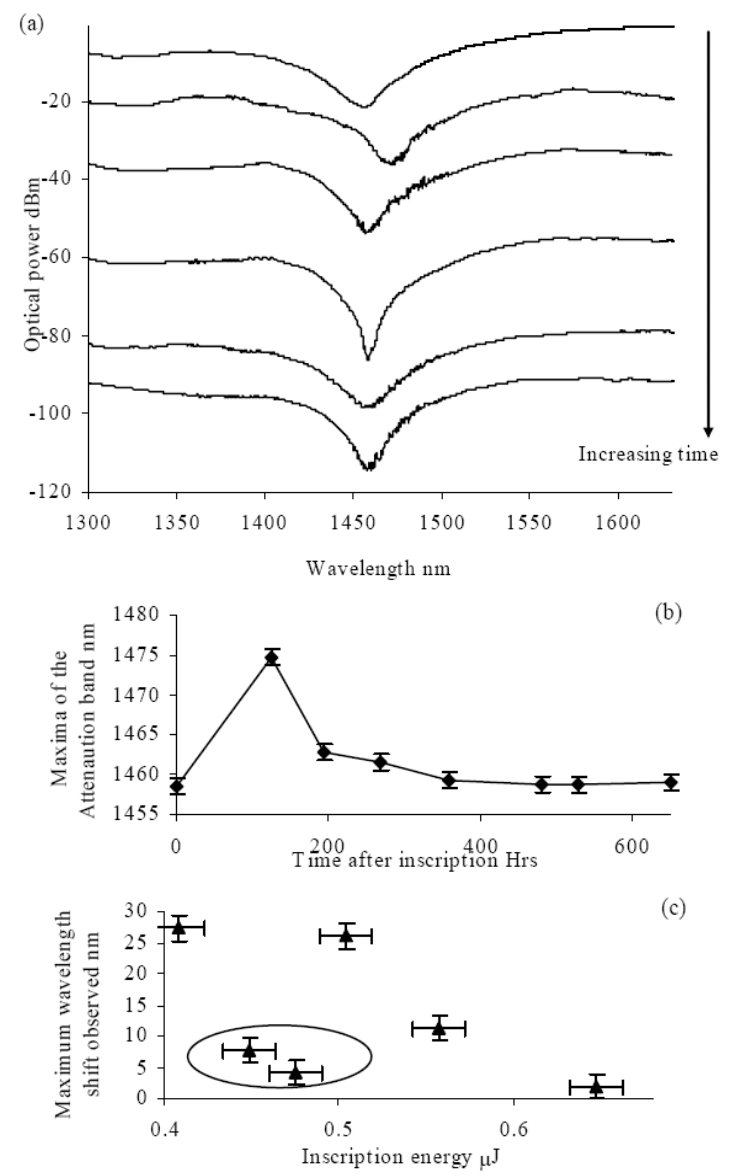

Fig. 10. (a)Typical example of the post-fabrication spectral evolution at room temperature of the transmission spectrum of a femtosecond inscribed LPG written in ESM PCF. (b) The spectral response of the LPG as a function time. (c) The maximum red wavelength shift observed with the LPGs fabricated in the ESM PCF as a function of inscription power. 

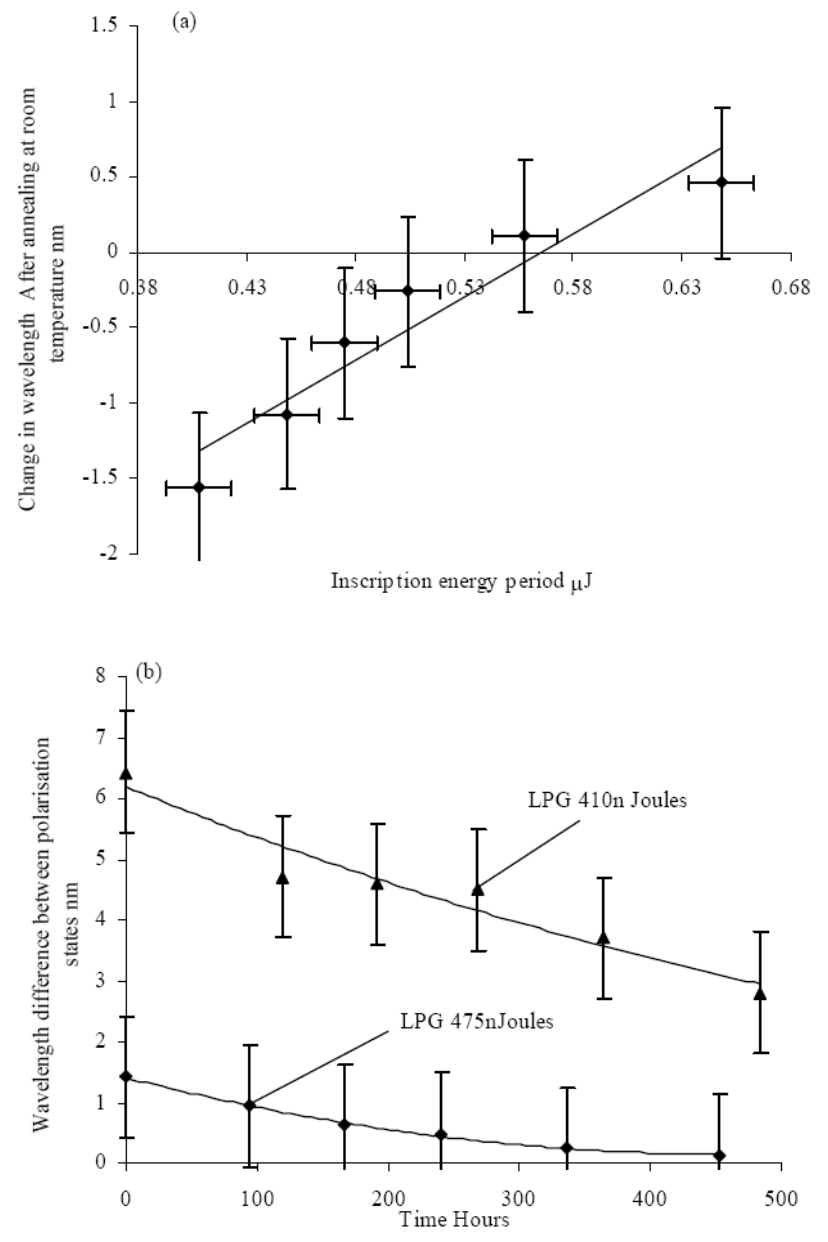

Fig. 11. (a) The resultant wavelength shift after annealing at room temperature (b) The change in polarisation dependency with annealing at room temperature.

\subsection{Annealing studies at $80_{0} \mathrm{C}$}

Dramatic changes were observed for the LPG annealed at $80^{\circ} \mathrm{C}$, both in wavelength shift, strength and profile of the attenuation bands, see figures $12 \mathrm{a}$ and $12 \mathrm{~b}$. The largest resultant post-annealing wavelength shift of $23 \mathrm{~nm}$ was observed with the lowest inscription energies for the LPGs, see figure 13a. The strengths of the LPGs vary with this annealing condition with the highest reduction in coupling also seen with the lowest inscription energies (an $8 \mathrm{~dB}$ reduction, see figure 13b). Also it was noted that the time taken to obtain a steady-state spectrum varied from 60 hours for the LPG inscribed with $475 \mathrm{~nJ}$ to 150 hours for the LPG inscribed with 650nJ. 

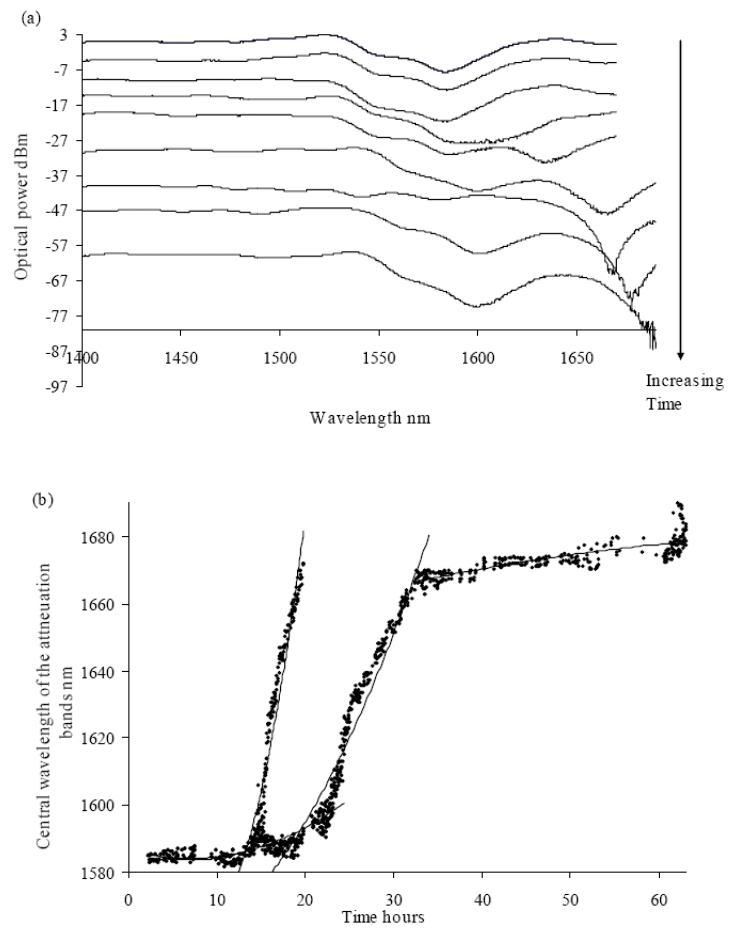

Fig. 12. Annealing at $80^{\circ} \mathrm{C}$.(a)The temporal evolution of the transmission spectrum of a LPG written in ESM PCF (period $400 \mu \mathrm{m}$, inscription energy $475 \mathrm{~nJ}$ ). (b) The spectral evolution during annealing of the central wavelength of the attenuation band of the LPG (period $400 \mu \mathrm{m}$, inscription energy $470 \mathrm{~nJ})$.
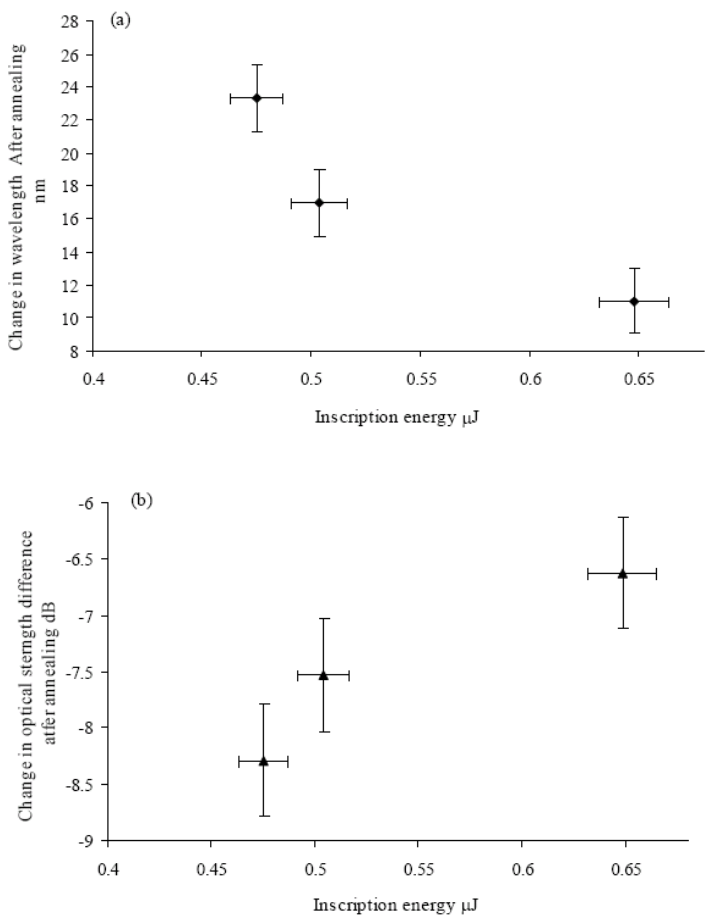

Fig. 13. (a) The resultant wavelength shift after annealing at $80{ }^{\circ} \mathrm{C}$ as a function of inscription energy. (a) The resultant change in optical strength of the attenuation band after annealing $80^{\circ} \mathrm{C}$ as a function of inscription energy. 


\section{CONCLUSIONS}

A series of LPGs was inscribed in photonic crystal fibre by a femtosecond laser system; when subjected to bending they were found to be spectrally sensitive to bend orientation, yielding red and blue wavelength shifts depending upon fibre curvature with a low temperature sensitivity. This suggests that these PCF devices have the potential to be applied as curvature sensors with high resolution. One of the main reasons for using femtosecond inscribed LPGs is their ability to operate at high temperatures 10 but some recent studies9 have suggested this may not be without problems. The current study has shown that the LPGs' thermal annealing properties are complex and intimately linked to their fabrication conditions, such as the inscription energy. This behaviour is manifested as post fabrication spectral evolution, both red and blue wavelength shifts at room temperature, depending upon inscription energy with the greatest shift observed, $1.5 \mathrm{~nm}$, corresponding with the lowest energy; 410nJ. The room temperature annealing results suggest that there is an optimum energy for minimal wavelength shift at room temperature; approximately $570 \mathrm{~nJ}$ for our arrangement. The results obtained so far for the higher annealing temperature $\left(80^{\circ} \mathrm{C}\right)$ have revealed red wavelength shifts, with the highest again occurring with the lowest inscription energy. The results so far suggest that greater understanding is required of the index modification mechanism of the LPGs to prevent misinterpretation of data from sensing applications.

\section{ACKNOWLEDGEMENT}

The authors acknowledge partial funding for this work from the Research Promotion Foundation Infrastructure Grant "FEMTO".

*kyriacos.kalli@cut.ac.cy; phone 35725002609

\section{REFERENCES}

1 D. Linde et al, "Breakdown threshold and plasma formation in femtosecond laser-solid interaction", JOSA. B, Vol. 13, No. 1, pp.216, 1996

2 A. Streltsov et al, "Study of femtosecond-laser-written waveguides in glasses", JOSA B, Vol. 19, No. 10, pp. 24962504,2002

3 A. Vengsarkar et al, "Long-Period Gratings as Band-Rejection Filters", J. Lightwave Technol., Vol.14, no.1, pp.58-64, 1996

4 V. Bhatia, A.M. Vengsarkar, "Optical fibre long-period grating sensors", Opt. Lett., Vol.21, no.9, pp.692-694, 1996

5 H.J. Patrick, A.D. Kersey, F. Bucholtz, "Analysis of the response of long period fiber gratings to external index of refraction", J. Lightwave Technol., Vol.16, no.9, pp.1606-1612, 1998

$6 \mathrm{~V}$. Bhatia, "Applications of long-period gratings to single and multi-parameter sensing", Opt. Express., Vol. 4, no. 11, pp.457-466, 1999

7 X. Shu et al, "Room-temperature operation of widely tunable loss filter", Electron. Letts. Vol. 37, no. 4, pp.216-218, 2001

8 T. Allsop et al, " Detection of Organic Aromatic compounds in Paraffin by a long period fiber grating optical sensor with optimised sensitivity", Opt. Commun., Vol.191, pp.181-190, 2001

9 H.J. Patrick, C.C. Chang, S.T. Vohra, "Long period fibre gratings for structural bend Sensing”, Electron. Letts., Vol. 34 , no. 18 , pp. 1773,1998

10 W. Du, H. Tam, M. Liu, X. Tao, "Long-period fiber grating bending sensors in laminated composite structures," in Sensory Phenomena and Measurement Instrumentation for Smart structures and materials. Proc. SPIE 3330, pp.284-292, 1998

11 H.J. Patrick, "Self-aligning, bipolar bend transducer based on long period grating written in eccentric core fibre", Electron. Letts., Vol. 36, no. 21, p. 1763-1764, 2000 
12 J. Petrovic et al, "Sensitivity of LPGs in PCFs Fabricated by an Electric Arc to Temperature, Strain, and External Refractive Index", J. Lightwave Technol, Vol.25, no.5, pp. 1306-1312

13 Y. Youk et al, "Refractive Index Profiling of a Core-Doped Photonic Crystal Fiber", Phot. Tech. Lett., Vol. 19, No. 11, pp.87, 2007

14 T. Wu et al, “A Novel Ultraflattened Dispersion Photonic Crystal Fiber”, Phot. Tech. Lett., Vol. 17, No. 1, pp.67, 2005

15 H. Dobb, K. Kalli, D. J. Webb, “Temperature-insensitive long period grating sensors in photonic crystal fibre”, Elec. Lett., Vol. 40, pp. 657- 658, 2004

16 K. Morishita, et al, "Fabrication and Resonance Wavelengths of Long-Period Gratings Written in a Pure-Silica Photonic Crystal Fiber by the Glass Structure Change", Lightwave Technol., Vol. 22, No. 2, pp.625, 2004

17 A. Martinez, et al, "Point by point FBG inscription by a focused NIR femtosecond laser", OSA Conf. Proc. CLEO/IQEC and PhAST Washington, DC, CMY6, 2004

$18 \mathrm{~T}$. Allsop, et al, "A comparison of the spectral properties of high temperature annealed long period gratings inscribed by fs laser, UV, and fusion arc", SPIE Vol 6193, Photonics Europe, Strasburg, France, 2006. 